\title{
Strontium Sr-89
}

National Cancer Institute

\section{Source}

National Cancer Institute. Strontium Sr-89. NCI Thesaurus. Code C1567.

A radioisotope of strontium, a heavy metal found naturally as a non-radioactive element. With a half-life of 50 days, strontium-89 is a beta emitter that is metabolized much like calcium. This agent is preferentially absorbed by osteoblastic bone metastases. (NCI04) 